\title{
Tropane alkaloids and terpenes synthase genes of Datura stramonium (Solanaceae)
}

\author{
Sabina Velázquez-Márquez ${ }^{1}$, Iván M De-la-Cruz ${ }^{1}$, Rosalinda Tapia-López ${ }^{1}$, Juan Núñez-Farfán ${ }^{\text {Corresp. } 1}$ \\ ${ }^{1}$ Laboratorio de Genética Ecológica y Evolución, Departamento de Ecología Evolutiva, Instituto de Ecología, Universidad Nacional Autónoma de México, \\ Ciudad de México, D F, MEXICO \\ Corresponding Author: Juan Núñez-Farfán \\ Email address: farfan@unam.mx
}

Background. Plants have evolved physical-chemical defense to prevent/diminish damage by their enemies. Chemical defense involves the synthesis' pathways of specialized toxic, repellent, or anti-nutritive metabolites to herbivores. Molecular evolutionary studies have revealed the origin of new genes, acquisition and functional diversification along time in different plant lineages. Methods. Using bioinformatic tools we analyze gene divergence of tropane alkaloids (TAs) and terpene synthases (TPSs) in Datura stramonium and other species of Solanaceae; compared gene and amino acids sequence of TAs and TPSs on genomes, cDNA and proteins sequences of Viridiplantae. We analyzed two recently assembled genomes of $D$. stramonium (Ticumán and Teotihucán), transcriptomes of Datura metel and genomes of other Solanaceae. Hence, we analyzed variation of TAs and TPSs to infer genes involved in plant defense and plant responses before stress. We analyzed protein modeling and molecular docking to predict interactions between $\mathrm{H} 6 \mathrm{H}$ and ligand; we translated the sequences (Teo19488, Tic8550 and Tic8549) obtained from the two genomes of $D$. stramonium by using Swiss-Model and Ramachandran plot and MolProbity structure validation of Teo19488 protein model. Results. For TAs, we detected an expansion event in the tropinone reductase II (TRII) and the ratio synonymous/nonsynonymous substitutions indicate positive selection. In contrast, a contraction event and negative selection was detected in tropinone reductase I (TRI). In Hyoscyamine 6 bhydroxylase $(\mathrm{H} 6 \mathrm{H})$, enzyme involved in the production of tropane alkaloids atropine and scopolamine, the synonymous/non-synonymous substitution ratio in its dominion indicates positive selection. For terpenes (TPS), we found 18 DsTPS in D. stramomiun and 7 in $D$. metel; evolutionary analyses detected positive selection in TPS10.1 and TPS10.2 of $D$. stramonium and $D$. metel. Comparison of copies of TPSs in $D$. stramonium detected variation among them in the binding site. Duplication events and differentiation of TAs and TPSs of $D$. stramonium, as compared to other Solanaceae, suggest their possible involvement on adaptive evolution of defense to herbivores. Protein modeling and docking 
show that the three protein structures obtained of DsH6H from Teo19488, Tic-8550 and Tic8549 maintain the same interactions and the union site of 20G-Fell_Oxy with the Hy-0 ligand as in 6TTM of $D$. metel. Conclusion. Our results indicate differences in the number of gene copies involved in the synthesis of tropane alkaloids, between the genomes of $D$. stramonium from two Mexican populations. More copies of genes related to the synthesis of tropane alkaloids (TRI, TRII, H6H, PMT-10) are found in D. stramoniumas compared to Viridiplantae. Likewise, for terpene synthases (TPS), TPS-10 is duplicated in D. stramonium and $D$. metel. Further studies should be directed to experimentally assess gain (overexpression) or loss (silencing) of function of duplicated genes. 
Tropane alkaloids and terpenes synthase genes of Datura stramonium (Solanaceae)

1

2

3 Sabina Velázquez-Márquez ${ }^{1}$, Iván. M. De-la-Cruz ${ }^{1}$, Rosalinda Tapia-López ${ }^{1}$ and Juan Núñez-

4 Farfán ${ }^{1 *}$

5

$6{ }^{1}$ Laboratorio de Genética Ecológica y Evolución, Departamento de Ecología Evolutiva, Instituto

7 de Ecología, Universidad Nacional Autónoma de México, Ciudad de México, D F, MEXICO

8

$9 *$ Corresponding Author:

10 Juan Núñez-Farfán ${ }^{1}$

11

12

13

14

15 


\section{ABSTRACT}

Background. Plants have evolved physical and chemical defense to prevent/diminish damage by their natural enemies. Chemical defense involves the synthesis' pathways of specialized toxic, repellent, or anti-nutritive metabolites to herbivores. Molecular evolutionary studies have revealed the origin of new genes, acquisition and functional diversification along time in different plant lineages. Here, using bioinformatic tools we analyze gene divergence of tropane alkaloids (TAs) and terpene synthases (TPSs) in Datura stramonium and other species of Solanaceae.

Methods. We compared the gene and amino acids sequence of TAs and TPSs by analyzing the genomes, cDNA and proteins sequences of species Viridiplantae. We analyzed two genomes of Datura stramonium (localities of Ticumán and Teotihucán), recently assembled in our lab, transcriptomes of Datura metel and the genomes of other species of Solanaceae. Hence, we analyzed the variation of TAs and TPSs to infer the genes involved in plant defense and plant responses before stress. In addition, we analyzed protein modeling and molecular docking to predict interactions between $\mathrm{H6H}$ and ligand; we translated the sequences (Teo19488, Tic8550 and Tic8549) obtained from the two genomes of $D$. stramonium by using Swiss-Model and Ramachandran plot and MolProbity structure validation of Teo19488 protein model.

Results. In the TAs' we detected an expansion event in the tropinone reductase II (TRII) and the ratio synonymous/non-synonymous substitutions indicate positive selection. In contrast, a contraction event and negative selection was detected in the tropinone reductase I (TRI). In Hyoscyamine $6 \beta$-hydroxylase $(\mathrm{H} 6 \mathrm{H})$, the enzyme involved in the production of the tropane alkaloids atropine and scopolamine, the synonymous/non-synonymous substitutions ratio in its dominion indicates positive selection. In the case of terpenes (TPS), responsible of inducible chemical defense in plants, we found 18 DsTPS of D. stramomiun and 7 in D. metel; evolutionary analyses detected positive selection in TPS10.1 and TPS10.2 of D. stramonium and D. metel. Further, comparison of copies of TPSs in D. stramonium detected variation among them in the binding site. Duplication events and differentiation of TAs and TPSs of $D$. stramonium, as compared to other solanaceous species, suggest their possible involvement on 
47 adaptive evolution of chemical defense against herbivores. Protein modeling and docking show 48 that the three protein structures obtained of DsH6H from Teo19488, Tic-8550 and Tic8549

49 maintain the same interactions and the union site of 2OG-FeII_Oxy with the Hy-o ligand as in

50 6TTM of Datura metel. Further studies should be directed to experimentally assess gain

51 (overexpression) or loss (silencing) of function of duplicated genes.

52

53 Conclusion. Our results indicate differences in the number of gene copies involved in the 54 synthesis of tropane alkaloids, between the genomes of Datura stramonium from the two 55 Mexican populations (Ticumán and Teotihuacán). Furthermore, more copies of genes related to 56 the synthesis of tropane alkaloids (TRI, TRII, H6H, PMT-10) are found in D. stramonium as 57 compared to Viridiplantae. Likewise, for terpene synthases (TPS), TPS-10 is duplicated in $D$.

58 stramonium and D. metel. These results point future experimental studies of gain/loss of 59 duplicated genes. 


\section{INTRODUCTION}

Plants are exposed to manifold environmental factors, biotic and abiotic, that affect their lifetime reproductive success. To cope with the different types of environmental stress, plants have evolved different mechanisms, ranging from morpho-anatomical, physiological, biochemical, molecular and epigenetic modifications, among others (Lamalakshmi et al., 2017). An ubiquitous defensive mechanism that protects plants from physical and biotic stresses is represented by the synthesis of specialized metabolites (SM; the so-called "secondary metabolites"). Thousands of chemical compounds found in plants promote protection against plants' natural enemies (i.e., pathogens, viruses and herbivores) (Mithöffer \& Boland, 2012). More than 40000 and 12000 terpenoids and alkaloids, respectively, have been described in plants (Zhou \& Pichersky, 2020). Tropane alkaloids (TAs) and Terpene synthases (TPSs) are important specialized molecules of plants that help to protect them from herbivores and pathogens (Kessler \& Baldwin, 2002). Tropane alkaloids are distinctive, but not exclusive, of Solanaceae (Wink, 2003; Mithöffer \& Boland, 2012; Pigatto et al., 2015) and in Datura stramonium, implicated in plant defense to herbivores (Shonle \& Bergelson, 2000; Castillo et al., 2014; Miranda-Pérez et al., 2016). Terpene synthases are enzymatic genes involved in the synthesis of volatile organic compounds (VOCs) in many organisms (Picazo-Aragonés et al., 2020). Alkaloids and VOCs negatively affect herbivores. The concentration of VOCs may even rise before herbivores start to feed (Heil et al., 2008). Recent molecular studies in plants indicate modifications in the expression of SM as defense mechanisms (Mansfield, 2000; Van Etten et al., 2001). Such modifications may result from the composition of the herbivore community, their abundance and dominance (Poelman \& Keizer, 2016). In this study we present a bioinformatic analysis of the changes in genes involved in the synthesis of tropane alkaloids and terpene synthases in Solanaceae, with particular attention to Datura stramonium. Gene modifications may include changes in the regulatory sequence or in the dominion, in the length of genes (including duplication), and post-transduction modifications (Defoort et al., 2019). Here, we analyze events of duplication in Solanaceae, with reference to whether duplicates are involved in modifications of biosynthetic pathways of TAs and TPSs.

Generally, copies of genes can have different fates. If different copies are retained, they may undergo sub-functionalization, dividing the original function. Alternatively, one copy may undergo neofunctionalization, acquiring a new function. However, the most frequent outcome is 
94 pseudogenization, whereby one copy becomes non-functional (pseudogene) and tends to 95 disappear due to accumulation of deleterious mutations. A pseudogene shows strong similarity to

96

97 98 the parental copy, which encodes a particular protein, but has one or more alterations such as premature stop codons, mutations that cause phase changes, deletions and/or insertions that prevent a protein from being functional $(\mathrm{Ohno}, 1970)$. It has been reported that in some species the retention of pseudogenes for a long time only accumulates neutral mutations. However, several questions arise regarding the survival mode of these inactivated genes (Ohno, 1970; Lynch \& Conery, 2003; Conant \& Wolfe, 2008; Wang et al., 2012). Some retained functions maintain developmental homeostasis, for instance, the ability to rapid respond to a wide variety of environmental cues (Wang et al., 2012). One first defense response of plants involves the activation of signaling mechanisms (De-La-Cruz et al., 2020), and therefore protein turnover. It is estimated that more than $80 \%$ of the proteins are degraded through the proteosome pathway (Maltsev, 1999). In recent years it has been reported that the responses of specialized metabolites are grouped into several multigenic families. Therefore, we aim to elucidate the evolutionary process of two gene families, TAs (Tropane alkaloids) and TPSs (terpenes synthases) (Karunanithi et al., 2019; De-La-Cruz et al., 2021). Studies conducted in Solanaceae have found rapid responses to biotic and abiotic stimuli, in a matter of minutes, of genes involved in the synthesis of specialized metabolites (atropine, scopolamine, VOCs) (Brille et al., 2019). To date, several groups have reported different TAs and TPSs and have found similar results (Bharat \& Sharma, 2015).

In this study we analyze the evolutionary history of genes involved in the synthesis of tropane alkaloids and terpene synthase in the Solanaceae family and specifically in draft genomes of two plants of Datura stramonium from populations of México (Ticumán and Teotihuacán). Overall, our bioinformatic analyses infer duplication of the genes of the main biosynthetic pathways leading to specialized metabolites.

\section{MATERIALS AND METHODS}

Selection and bioinformatic analyses: Identification of TAs and TPs, genomics, transcripts and protein sequences

We obtained the sequences of nucleotide, transcripts and proteins of TAs and TPSs. Nine genomes (Table 1) of Solanaceae species were sourced for protein coding genes and CDS 
127 (Coding Sequence) genomes from the Sol Genomics Network (see links in Table 1): Nicotiana

128 tabacum (Edwards et al. 2017), Nicotiana sylvestris (Sierro et al. 2013), Nicotiana attenuata (Xu

129 et al. 2017), Nicotiana tomentosiformis (Sierro et al. 2013), Solanum pimpinellifolium (Razali et

130 al. 2018), Solanum lycopersicum (The Tomato Genome Consortium, 2012), Solanum pennellii

131 (Bolger et al. 2014 b), Solanum tuberosum (Xu et al. 2011), Capsicum annuum, CM334 v1.55

132 (Kim et al. 2014). In addition, data of Datura stramonium were extracted from protein whereas

133 TAs and TPSs' sequence were obtained from the genome of $D$. stramonium from the Mexican

134 populations of Ticumán, Morelos (1844'28.19"N, 99 7'44.26"W), and Teotihuacán, Estado de

135 México (1941'16.58"N, 9850'4.14"W) (https://github.com/icruz1989/Datura-stramonium-

136 genome-project) (De-la-Cruz et al., 2021) and transcriptomes (https://medplantrnaseq.org/).

137 We downloaded the whole genome of D. stramonium to extract the TPSs genes and gene

138 prediction was performed using AUGUSTUS (Stanke et al., 2006). All sequences were uploaded

139 and mapped using as reference species of Solanaceae. A total of 366000 genes were extracted,

140 and gene redundancy was reduced with CDHIT (Chen et al., 2016). The selected TPSs genes

141 were blasted against the NCBI, UNIPROT databases and the nine genomes of Solanaceae, using

142 a threshold value of $1 \mathrm{E}^{-5}$. All TPSs were downloaded from Viridiplantae followed by a protein-

143 protein blast; hits above $80 \%$ of similarity and $70 \%$ of cover were saved for further analyses.

144 Meta-alignments of protein sequences were performed using T-coffee (Wallace et al., 2006),

145 MUSCLE v3.8 (Edgar, 2004) and MAFF (https://mafft.cbrc.jp/alignment/server/) v6. (Rowicki

146 et al., 2019).

147

148 Identification of orthogroups and inference of phylogenetic tree of TPa and TPSs.

149 Construction of orthogroups, gene families, was performed according to De-la-Cruz et al. (2021)

150 using Orthofinder v2.3.3 (Emms \& Kelly 2015, 2019). The phylogenetic tree inference was

151 accomplished using the program BEAST with the pair-wise deletion option, and reliability of the

152 obtained phylogenetic tree was tested by bootstrapping with 1000,000 MKKM chains. The

153 model of substitution selected was JTT $+\mathrm{G}+\mathrm{F}$, with a branch support of 1 (Drummond et al.,

154 2002). The trees were edited at the interactive tree of life (https://itol.embl.de) (Letunic \& Bork, 155 2019). 
156 We downloaded all orthologous sequences of TAs and TPSs of D. stramonium, D. metel and

157 nine species of Solanaceae (see Table 1) to construct the orthogroups. Downloaded sequences of

158 proteins and ORFs were extracted using TransDecoder and corroborated with GenScan. TPS

159 genes were translated with GenScan and checked for the right direction and correct CDS; we

160 corroborated this with the NCBI. The database used for BLAST was Uniprot; all available data

161 for the analyzed plant species were downloaded. These amounts 847,544 sequences of TAs and

162 TPSs. BLAST was carried out with a filtering E-value $<1 \mathrm{e}^{-5}$ and only hits above $85 \%$ were

163 selected (Data S1, S2). Orthogroups were inferred using Orthfinder v 2.3.3. DIAMONS (Emms

$164 \&$ Kelly 2015, 2019) while OrthoMCL was used to assess orthologs among species (Chen et al., 165 2006).

166

167 Protein modeling

168 Molecular modelling and protein-ligand docking was performed with GLIDE software

169 (Schrödinger Release 2021-1: Glide, Schrödinger, LLC, New York, NY, 2021). Simulations of 170 interactions for each protein was done in a quadrat of $6 \mathrm{LU} /$ processed with the same coordinates 171 of the crystalized ligand as described in Gutierrez et al. (2020).

172

173

174

175

176

177

178

179

180

181

182

183

184

185

The SWISS-MODEL (http://www.expasy.org/swissmod/SWISS-MODEL.html)

(Waterhouse et al., 2018) was employed to model the tertiary structure of proteins, from the previously determined structure by the dominion DIOX_N (PF14226) (Hagel et al., 2010) as a blueprint of modelling. Models were visualized and analyzed through Visual Molecular Dynamics (Waterhouse et al., 2018; Bienert et al., 2017)

\section{Molecular Docking}

The in-silico docking (AutodockVina) of the PDB files (produced by the SWISS-Model) of Tic8550 (Ticumán) and Teo19488 (Teotihuacán) was performed in a simulation of molecular coupling with the native ligand of $\mathrm{H} 6 \mathrm{H}$, which is Hyoscyamine (Hy-o). For docking, the protein was prepared with AutodockTools, assigning the parameters of energies and force fields of AutoDock4.0. Then, we obtained the results of the thermodynamically favored conformation for the Hy-o ligand using the algorithm VINA. The selected conformation had affinity coupling parameters of -7.3 and $-7.5 \mathrm{kcl} / \mathrm{mol}$ for Teo19488 and Tic8550, respectively. As positive control, 
186 we performed the re-docking of the molecule of reference 6TTM (Datura metel), eliminating the

187 ligand of the crystallized structure; the adopted conformation is similar to that obtained by the 188 method of crystallization, and the coupling affinity for the reference model was $-7.9 \mathrm{kcal} / \mathrm{mol}$.

189 The produced models are theoretically correct, and it is predicted that these have an

190 oxidoreductase and use Hy-o as substrate.

191

192

Tropane alkaloids

193

194

195

196

197

198

199

200

201

202

203

204

205

206

207

208

209

210

211

Analyses detected variation in the number of TAs gene copies in D. stramonium: DsTRI, DsTRII, DsPMT and DsH6H. Compared to Viridiplantae D. stramonium has one or more copies of these genes suggesting a genome expansion of TAs.

We detected eight copies of tropinone reductase I (DsTRI) and four copies of DsTRII. TRI and TRII define two groups in Solanaceae (Fig. 1A). The gene DsTRII have had duplications events in D. stramonium (Fig. 1B). The first duplication occurs in Rosidae-Asteridae, followed by three duplications at the base of Solanaceae and one more in D. stramonium (Fig. 1B). The phylogeny and structure 32 of protein sequences (TRI) from different plant species show variability in the ADH_Short_C2 TRI dominion (Fig. 2); in D. stramonium (Tic23_dati33027) there a duplication of this dominion but shorter (Fig. 2).

In Hyoscyamine- 6-ß-hydroxylase $(\mathrm{DsH} H \mathrm{H})$ in the Ticumán genome we detected two copies of the gene, Tic8550 and Tic8549 (Fig. 3A). Tic 8550 has a tandem duplication of the DIOX-N dominion of 79 amino acids (Fig. 3) whereas Tic8549 has the dominion 2oxaglutarate (65 amino acids) (Fig. 3A). In contrast, in the Teotihuacán genome, DsH6H (i.e., Teo19488) has only one copy (Fig. 3). The alignment of 29 sequences of H6H of different plant species indicate the variation in the duplicated DIOX-N dominion in Tic8550 (Fig. 3B).

In N-methyl putremescine transferase (DsPMT) we found variation in the number of gene copies in comparison to other Solanaceae (Fig. 4). In Tucumán's genome there are three copies while two copies were detected in Teotihuacán's genome (Fig. 4, Suppl. Fig. 1). 
213

214

215

216

217

218

219

220

221

222

223

224

225

226

227

228

229

230

231

232

233

234

235

236

237

238

239

240

241

Eighteen TPSs genes were found in the genomes of D. stramonium (Fig. 5; Table S1) and seven in Datura metel (Fig. S2). The 18 DsTPS found in D. stramonium are distributed in four subfamilies, identified for other angiosperms: Nine in TPSa, 3 in TPSb, 2 in TPSc, 2 in TPSg and 2 TPS- unknown (Fig. S3, Table S1 and S2) (Huang, 2013, 2017; Falara et al., 2011).

The domains of these TPSs, directly involved in the biosynthesis of terpenoids, show expansion events and positive selection in TPS10 (Fig. 6; Table S3). Changes in the dominions are: Terpene synthase, N-terminal domain (IPR001906), Terpenoid cyclases/protein prenyl transferase alpha-alpha toroid (IPR008930), Terpene synthase, metal-binding domain (IPR005630), Terpene cyclase-like 1, C-terminal (IPR034741). Analyses indicate that Solanaceae have the gene TPS10.1 whereas only D. stramonium and D. metel possess TPS 10.2 (Fig. 7 and Table S2 and S3,); this possibly is a duplicate of TPS10.1_like.

\section{Protein modeling and molecular docking}

Superimposition of the reported protein structure of H6H from D. metel (PDB ID 6TTM) with the corresponding model obtained from $\mathrm{DsH} 6 \mathrm{H}$ sequences for the two genomes of $D$. stramonium from two Mexican populations (Teo19488 and Tic8550), indicate similarities (Fig. 7A). The Teo19488 predicted structure match very well with the DmH6H structure (Fig. Suppl. 2); similarly, Tic8550 matches well except for some residues that were not modeled (Fig. 7B, Fig. Suppl 3). This fragment of Tic8550 have a big terminal protein sequence (NQTMMLKWLLFLKLVYKQYLYKLYSTIGEKAEKDVINHGVPEKIMVEAMEFTKSFL HCLLRKKKSLSQKEASIKQSSML) of 79 amino acids was not modeled (Fig. 7B, cf. Fig. 3B). Further, a NCBI protein-protein blast of this sequence aligns well to the region: 33-72 of DmH6H fragment; we analyzed this fragment separately as s Tic8550_Fragment (Fig.86). The Tic8549 sequence corresponds to a 65 amino acids sequence (MLPIIPRPKSTLGAGGHYDGNIITFLQQDCLACNNSLLRMTNGLLLNLSYCFCGLSGT HSKGYEQ) that was modeled as hyoscyamine 6 beta-hydroxylase like fragment (Fig. 8). This fragment (Tic8549) aligns to DmH6H in one section of the binding pocket, where His-217 and Asp-219 are coordinated with $\mathrm{Ni}^{2+}$ (Fig. 8C). The presence of these amino acids in Tic8549 
242

243

244

245

246

247

248

249

250

251

252

253

254

255

256

257

258

259

260

261

262

263

264

265

266

267

268

269

270

sequence is interesting since $\mathrm{Ni}^{2+}$ ion, a surrogate of the natively present $\mathrm{Fe}^{2+}$ ion, is coordinated by the side chains of His-217 (strand $\beta \mathrm{II}$ ), Asp-219 (loop $\beta \mathrm{II} / \beta \mathrm{III}$ ) and His-274 (strand $\beta \mathrm{VII}$ ) that forms a metal binding His-X-Asp...His motif, highly conserved in the oxoglutarate dependent oxygenases (ODD) family (Kluza et al., 2020).

Protein models of $\mathrm{H6H}$ for D. metel and D. stramonium (Teo19488 and Tic8550) predict more interaction between residues in Tic8550 than on D. metel and Teo19488 (Fig. 9). It has recently been reported that the binding pocket (BP) of $\mathrm{DmH6H}$ is mainly formed by hydrophobic amino acids. It has been demonstrated that in the crystalized $\mathrm{H} 6 \mathrm{H}$ the phenyl ring of Hyo is bounded in an aromatic cage formed by Phe-103, Tyr-295, Tyr-319, Phe-322 and Tyr-326, of which the most prominent bound is with Tyr-326 which forms $\mathrm{CH}-\pi$ hydrogen bonds with the phenyl ring of Hyo in an edge-to-face bidentate manner (Kluza et al., 2020) (Fig. 9A, D). Our results shown that the predicted model for Teo19488 have the same amino acids forming the aromatic cage composed by Phe-88, Tyr-255, Tyr-279, Phe-282 and Tyr-286 and the predicted pose of Hyo obtained by the docking in silico shows that the phenyl ring is inside of this aromatic cage (Fig. 9B). Nevertheless, the main interaction with Tyr-286 was not detected (Fig. 9E). This could be because the cutoff distance was set $4 \AA$ and the predicted pose of this residue is beyond this cutoff distance. The predicted structure of Tic8550 has different amino acids arrangement inside the binding pocket. This predicted structure does not contain the aromatic cage, instead it is predicted that the phenyl ring of Hyo inside of a cavity formed by the non-polar amino acids Leu-308, Leu-92, Ile-179, Met-181, Met-292 and the polar uncharged amino acids Asn-206 and Asn-289 (Fig. 9C). Also, it is predicted that the phenyl ring has several non-polar interactions with these side chain amino acids (Fig. 9F). The Asn206 forms a conventional hydrogen bond with the hydroxyl group from Hyo which may stabilize the structure in the BP.

\section{DISCUSSION}


271 Gene duplicates product of molecular evolution are the raw material for evolutionary innovation

272 (Defoort et al., 2019). Recent findings have uncovered the interactions and contrasting functions

273 of genes that may help plants to confront environmental stresses in nature and serve as important

274 agronomic characteristics. Hence, to trace the origin, molecular mechanisms, evolutionary fate

275 and function of gene duplicates is a main goal (Panchy et al., 2016; Soltis \& Soltis 2016; Van de

276 Peer et al., 2017). The phylogenetic analyses of genes involved in the synthesis of tropane

277 alkaloids (DsPMT, DsTRI, DsTrII and DsH6H) here presented, indicate that two genes (DsTrII

278 and DsH6H) have expanded in D. stramonium, presenting five and two copies, respectively.

279 Previous studies have shown that tropinone reductases are involved in the bifurcation of the

280 synthesis pathways in Solanaceae (Dräger, et al., 1992; Nakajima 1993; Kanehisa \& Sato, 2019).

281 These genes are responsive to environmental stimuli and activate the signaling pathways (Tian et

282 al., 2016; Campos et al., 2014). The presence of these genes in the Solanaceae, as revealed by

283 phylogenetic analyses, suggests that these duplicates in D. stramonium may contribute to

284 increase the production of tropane alkaloids. The absence of TRII in Zea mays and A. thaliana

285 suggest that these species never had this gene or lost it during their evolution. However, the gene

286 is also absent in species of other clades (e.gr., Brassica rapa, Glycine max, Vitis vinifera, etc.;

287 Fig. 1). Another possibility is that TRII is not related to the synthesis of tropane alkaloids in

288 these species (Miller, 1999).

289 The gene DsPMT of D. stramonium found in the population of Ticumán, México, had an

290 extra domain of spermine-synthase in comparison with its homologous gene found in the same

291 species but from the population of Teotihuacán, México (De-la-Cruz et al., 2021). We named

292 this gene as DsPMT7568ti (Fig. 4), Spermine gene is a potent plant defense activator, with

293 protective effects of broad-spectrum (Seifi et al., 2019). Reports indicate that overexpression of

294 spermidine synthase enhances tolerance to multiple environmental stresses, including the attack

295 by herbivores and pathogens (Kasukabe et al., 2004). In addition, PMT is a key enzyme in the

296 catalysis of N-methylputrescine from putrescine and S-adenosyl-L-methionine and triggers the

297 production of hygrine and other different tropane alkaloids (Kanehisa \& Sato, 2019).

298 In contrast to other plants, in Solanaceae (Atropa belladona, Hyosciamus niger and

299 Datura stramonium), TRII is duplicated and under positive selection. In this line, we hypothesize

300 that TRII may have acquired a different functional importance after its duplication in $D$.

301 stramonium and hence, it has retained multiple copies of the gene. Yet, this hypothesis warrants 
302 further analysis. The phylogeny indicates an independent history, for instance, from

303 Brassicaceae.

304 Perhaps TRs in Brassicales have other functions, different to the production of alkaloids,

305 but using the same pathways. For instance, they may have conferred plasticity to deal with

306 environmental stresses. Plant species with small genome size may have lost considerable

307 fractions of their genome. Thus, enzymes with similar function may have been replaced by TRII.

308 Of the four subfamilies of TPSs found in the D. stramonium's genome, three have been reported

309 in other angiosperms (TPSa, TPSb and TPSg). TPS10 is member of the subfamily $a$ and its role

310 in defense against pathogens and herbivores has been dilucidated in Zea mays (Huang et al.,

311 2017; Tholl, 2006). We detected positive selection on TPS; positive selection on TPS10.1 and

312 TPS10.2 was detected in D. stramonium and D. metel. We speculate that the presence of two

313 copies of this gene can contribute to plant defense against plants' natural enemies (Köllner et al.,

$3142009,2020)$.

315 In this line, duplicated genes involved in the specialized metabolism and plant defense

316 often show differential restrictions (constraints, trade-offs, limitations) either indicating positive

317 selection or differential selection (Cotton et al., 2006). Apparently, TRII displays genic

318 redundancy since it possesses different copies and polymorphic sites. However, this may not be

319 the case since this process is only present in genes of primary metabolism or development

320 (Cotton et al., 2006; Maltsev et al., 2005) which are constitutive and display low variation. The

321 gene $\mathrm{H6H}$ is involved in the last step in the production of scopolamine and atropine in one of the

322 pathways for the production of tropane alkaloids (Kanehisa \& Sato 2019). Here, we detected one

323 duplicated in tandem of this gene in D. stramonium (Ticumán has two domains of DIOX_N

324 (PF14226), although each copy belongs to two different gene families (OG0028637 and

325 OG0043057; cf. Fig. 3). Similarly, we found 18 TPSs in D. stramonium that likely are involved

326 in plant defense against herbivores like in other plants species (e.gr., Bharat \& Sharma 2015).

327 Lineages in the Solanaceae have high values of the ratio $\mathrm{Ka} / \mathrm{Ks}$ (Table 2). Thus, positive

328 selection may have acted on TPS10.1 and TPS10.2 of D. stramonium, not recently and the

329 selection effect on allelic diversity may be masked by the accumulation of neutral mutation.

330 On the other hand, results of protein simulation and molecular docking suggest that the

331 structure and interactions of this protein $(\mathrm{H} 6 \mathrm{H})$ in Tic8550 might have a different structure to 
332 that of the known $\mathrm{H} 6 \mathrm{H}$; we speculate if this may be related to enhancing the efficiency of

333 alkaloids' synthesis.

334 It is interesting to note that the Tic8549 possess only the fragment 2OG-FeII_Oxy, 335 PF03171) whereas Tic8550 possess an extra dominion (DIOX_N) which aligns structurally to a 336 highly conserved region of $\mathrm{H} 6 \mathrm{H}$ proteins (Fig. S2 and Fig. S3) (and associated to a coordination 337 of binding metal $\mathrm{Ni}^{+2}$ (Fig. S5). Further, it is interesting noting that a particular gene Tic8549 is 338 associated to this fragment DIOX_N (PF14226).

339 Finally, the number of copies and differentiation of TAs and TPSs of D. stramonium, as 340 compared to other solanaceous species, suggest their involvement on adaptive evolution of 341 chemical defense against herbivores. Further studies should be directed to experimentally assess 342 gain (overexpression) or loss (silencing) of function of duplicated genes.

\section{CONCLUSION}

Our results indicate differences in the number of gene copies involved in the synthesis of tropane alkaloids, between the genomes of Datura stramonium from the two Mexican populations

348 (Ticumán and Teotihuacán). Furthermore, more copies of genes related to the synthesis of tropane alkaloids (TRI, TRII, H6H, PMT-10) are found in D. stramonium as compared to Viridiplantae. Likewise, for terpene synthases (TPS), TPS-10 is duplicated in D. stramonium and D. metel. These results point future experimental studies of gain/loss of duplicated genes.

352

353

Links to data on Figshare (Data S3)

\section{ACKNOWLEDGEMENTS}

355

356

357 358 359

360 361
We are grateful to the three reviewers whose comments and suggestions improved this contribution. We thank to Tonatiuh Campos García his advice in protein modelling. We appreciate the strong support from CONACyT and DGAPA UNAM. To the members of the Laboratory of Ecological Genetics and Evolution for encouragement and help.

\section{REFERENCES}


362 Bailey TL, Williams N, Misleh C, Li WW. 2006. MEME: discovering and analyzing DNA and

363 protein sequence motifs. Nucleic Acids Research 34(2):W369-W373 DOI: 10.1093/nar/gk1198.

364

365

Bienert, S, Waterhouse A, de Beer TAP, Tauriello G, Studer G, Bordoli L, Schwede T. 2017.

366

367

The SWISS-MODEL Repository - new features and functionality Nucleic Acids Research

45(D1): D313-D319 DOI:10.1093/nar/gkw1132.

368

369

Birgit D. 2006. Tropinone reductases, enzymes at the branch point of tropane alkaloid

370

metabolism. Phytochemistry 67(4):327-337 DOI: 10.1016/j.phytochem.2005.12.001.

371

Bolger A, Scossa F, Bolger M E, Lanz C, Maumus F, Tohge T, Quesneville H, Saleh Alseekh S,

372

Sørensen I, Lichtenstein G, Fich E A, Conte M, Keller H, Schneeberger K, Schwacke R, Ofner I,

Vrebalov J, Xu Y, Osorio S, Alves Aflitos S, Schijlen E, Jiménez-Goméz J M, Ryngajllo M,

Kimura S, Kumar R, Koenig D, Headland L R, Maloof J N, Sinha N, van Ham R C H, Klein

Lankhorst R M, Mao L, Vogel A, Arsova B, Panstruga R, Fei Z, Rose J K C, Zamir D, Carrari F,

Giovannoni J J, Weigel D, Usadel B, Fernie A R. 2014 The genome of the stress-tolerant wild

tomato species Solanum pennellii. Nature Genetics 46:1034-1038 DOI: 10.1038/ng.3046.

Brille F, Loreto F, Baccelli I. 2019. Exploiting plant Volatile Organic Compounds (VOCs) in agriculture to improve sustainable defense strategies and productivity of crops. Frontiers in

Plant Science 10: 264 DOI: 10.3389/fpls.2019.00264.

382

383

Campos ML, Kang JH, Howe GA. 2014. Jasmonate-triggered plant immunity. Journal of

Chemical Ecology, 40(7):657-675 DOI 10.1007/s10886-014-0468-3.

Castillo G, Cruz LL, Tapia-López R, Olmedo-Vicente E, Carmona D, Anaya-Lang AL, Fornoni

J, Andraca-Gómez G, Valverde PL, Núñez-Farfán J. 2014. Selection mosaic exerted by specialist and generalist herbivores on chemical and physical defense of Datura stramonium. PLoS ONE 9(7): e102478 DOI: 10.1371/journal.pone.0102478. 
391 Chen F, Mackey AJ, Stoeckert CJ, Roos D. 2006. OrthoMCL-DB: querying a comprehensive 392 multi-species collection of ortholog groups. Nucleic Acids Research 34:363-368.

393 https://doi.org/10.1093/nar/gkj123

394

395

Chen Q, Wan Y, Lei Y, Zobel J, Verspoor K. 2016. Evaluation of CD-HIT for constructing non396 redundant databases IEEE International Conference on Bioinformatics and Biomedicine (BIBM) 397 pp. 703-706 DOI: 10.1109/BIBM.2016.7822604.

398

399

Conan GC, Wolfe KH. 2008. Turning a hobby into a job: How duplicated genes find new 400 functions. Nature Reviews Genetics 9:938-950 DOI: 10.1038/nrg2482.

401

402

Cotton JA, Page RDM. 2006. Multiple mechanisms promote the retained expression of gene 403 duplicates in the tetraploid frog Xenopus laevis. PLoS Genetics 2(4):e56 DOI:

404 10.1371/journal.pgen.0020056.

405

406

Defoort J, Van de Peer Y, Carretero-Paule L. 2019. The Evolution of gene duplicates in 407 angiosperms and the impact of protein-protein interactions and the mechanism of duplication. 408 Genome Biology and Evolution 11(4):2292-2305 DOI: 10.1093/gbe/evz156.

409

De-la-Cruz I M, Hallab, A, Olivares-Pinto U, Tapia-López R, Velázquez-Márquez S, Piñero D, 411 Oyama K, Usadel B, Núñez-Farfán, J. 2021. Genomic signatures of the evolution of defence against its natural enemies in the poisonous and medicinal plant Datura stramonium (Solanaceae). Scientific Reports 11(1):1-19. https://doi.org/10.1038/s41598-020-79194-1

De-la-Cruz I.M., Velázquez-Márquez S., Núñez-Farfán J. 2020. What do we know about the 416 genetic basis of plant defensive responses to herbivores? A minireview. In: Núñez-Farfán J., 417 Valverde P. (eds) Evolutionary Ecology of Plant-Herbivore Interaction. Springer 
420 Dräger B, Portsteffen A, Schaal A, McCabe P, Peerless A. 1992. Levels of tropinone- reductase 421 activities influence the spectrum of tropane esters found in transformed root cultures of Datura 422 stramonium (L.). Planta 188(4):81-586 DOI: 10.1007/BF00197052.

423

424 Drummond AJ, Nicholls GK, Rodrigo AG, Solomon W. 2002. Estimating mutation parameters, 425 population history and genealogy simultaneously from temporally spaced sequence data.

426 Genetics 161:1307-132.

427

428 Edgar RC. 2004. MUSCLE: multiple sequence alignment with high accuracy and high 429 throughput. Nucleic Acids Research 32(5):1792-7 DOI: 10.1093/nar/gkh340.

431 Edwards KD, Fernandez-Pozo N, Drake-Stowe K, Humphry M, Evans AD, Bombarely A, Allen

432 F, Hurts R, White B, Kernodle SP, Bromley J, Sanchez-Tamburrino J, Lewis R, Mueller L. 2017.

433 A reference genome for Nicotiana tabacum enables map-based cloning of homologous loci

434 implicated in nitrogen utilization efficiency. BMC Genomics 8:448 DOI: 10.1186/s 12864-017435 3791-6.

436

437 Emms DM, Kelly S. 2015. OrthoFinder: solving fundamental biases in whole genome 438 comparisons dramatically improves orthogroup inference accuracy Genome Biology 16,157 DOI: 10.1186/s13059-015-0721-2.

440

441

Emms DM, Kelly S. 2019. OrthoFinder: phylogenetic orthology inference for comparative 442 genomics. Genome Biology 20:238 DOI: 10.1186/s13059-019-1832-y.

Falara V, Akhtar TA, Thuong TH, Spyropoulou EA, Bleeker PM, Schauvinhold I, Matsuba Y, Synthase Gene Family. Plant Physiology 157:770-789 DOI: 10.1104/pp.111.179648.

Hagel JM \& Facchini PJ. 2010. Dioxygenases catalyze the O-demethylation steps of morphine biosynthesis in opium poppy. Nature Chemical Biology 6:273 DOI:10.1038/nchembio.317. 
451 Heil M, Lion U, Boland W. 2008. Defense-Inducing Volatiles: In search of the active motif.

452 Journal of Chemical Ecology 34(5):601-604 DOI: 10.1007/s10886-008-9464-9.

453

454 Huang AC, Kautsar SA, Hong YJ, Medema MH, Bond AD, Tantillo DJ and Osbourn A. 2017.

455 Unearthing a sesterterpene biosynthetic repertoire in the Brassicaceae through genome mining

456 reveals convergent evolution. Proceedings of the National Academy of Sciences USA .

457 114:E6005-E6014 DOI: 10.1073/pnas.1705567114

458

459

Huang X, Xiao Y, Köllner T G, Zhang W, Wu J, Wu J, Zhang Y. 2013. Identification and

460

characterization of (E)- $\beta$-caryophyllene synthase and $\alpha / \beta$-pinene synthase potentially involved in

461

constitutive and herbivore-induced terpene formation in cotton. Plant Physiology and

462

Biochemistry 73:302-308 DOI: 10.1016/j.plaphy.2013.10.017.

463

Kanehisa M, Sato Y. 2019. KEGG Mapper for inferring cellular functions from protein

464

sequences. Protein Science 29:28-35 DOI: 10.1002/pro.3711.

465

466

Kasukabe Y, He L, Nada K, Misawa S, Ihara I, Tachibana S. 2004. Overexpression of

467

spermidine synthase enhances tolerance to multiple environmental stresses and up-regulates the

468

expression of various stress-regulated genes in transgenic Arabidopsis thaliana. Plant \& Cell

469

Physiology 45:712-22 DOI: 10.1093/pcp/pch083.

470

471

472

Kessler A., Baldwin I. 2002. Plant responses to insect herbivory: the emerging molecular analysis. Annual Review of Plant Biology 53: 99-328 DOI: 10.1126/science.291.5511.2141.

473

474 Kluza A, Wojdyla Z, Mrugala B, Kurpiewska K, Porebski PJ, Niedzialkowska E, Borowski T.

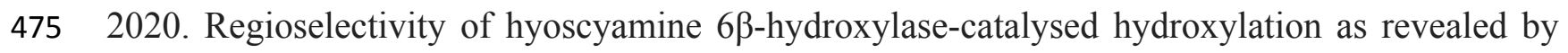

476 high-resolution structural information and QM/MM calculations. Dalton Transactions 49:4454-

4774469 DOI: 10.1039/d0dt00302f.

478

479 Köllner TG, Degenhardt J \& Gershenzon J. 2020. The product specificities of maize terpene

480 synthases TPS4 and TPS10 are determined both by active site amino acids and residues adjacent 481 to the active site. Plants 9:552 DOI:10.3390/plants9050552. 
483 Köllner TG, Gershenzon J \& Degenhardt J. 2009. Molecular and biochemical evolution of maize

484

485

486

487

488

489

490

491

492

493

494

495

496

497

498

499

500

501

502

503

504

505

506

507

508

509

510

511

512

terpene synthase 10, an enzyme of indirect defense. Phytochemistry 70:1139 1145 DOI:

10.1016/j.phytochem.2009.06.011.

Kurananithi PS \& Zerbe P. 2019._Terpene Synthases as Metabolic Gatekeepers in the Evolution of Plant Terpenoid Chemical Diversity. Frontiers in Plant Science 10:1166 DOI:

10.3389/fpls.2019.01166

Lamalakshmi E, Kumar S, Basanta T, Susheel S. Beemrote A, Chingakham P, Chongtham SK, Chongtham HS, Yumlembam R, Haribhushan A, Prakash N, Wani S.H .2017. Adaptation strategies and defence mechanisms of plants during environmental stress. In: Ghorbanpour M, Varma A (eds), Medicinal Plants and Environmental Challenges. pp. 359-413 Springer DOI: org/10.1007/978-3-319-68717-9_20.

Larkin MA, Blackshields G, Brown NP, Chenna R, McGettigan PA, McWilliam H, Valentin F, Wallace IM, Wilm A, Lopez R, Thompson JD, Gibson TJ, Higgins DG. 2007. Clustal W and Clustal X version 2.0. Bioinformatics 23:2947-2948 DOI: 10.1093/bioinformatics/btm404. Letunic I \& Bork P. 2019. Interactive Tree of Life (iTOL) v4: recent updates and new developments. Nucleic Acids Research 47:256-259 DOI: 10.1093/nar/gkz239.

Lynch M \& Conery JS. 2003. The origins of genome complexity. Science 5649(302):1401-1409 DOI: $10.1126 /$ science. 1089370 .

Maltsev N, Glass EM, Ovchinnikova G, Gu Z. 2005. Molecular mechanisms involved in robustness of yeast central metabolism against null mutation. Journal of Biochemistry 34(5):601-604. DOI: 10.1093/jb/mvi017.

Miller JD, Arteca RN, Pell EJ. 1999. Senescence-associated gene expression during ozoneinduced leaf senescence in Arabidopsis. Plant Physiology 120:1015-1024 DOI: 10.1104/pp.120.4.1015.

PeerJ reviewing PDF | (2020:02:45794:1:2:NEW 15 Apr 2021) 
513

514 Miranda-Pérez A, Castillo G, Hernández-Cumplido J, Valverde PL, Borbolla M, Cruz LL,

515 Tapia-López R, Fornoni J, Flores-Ortiz CM, Núñez-Farfán J. 2016. Natural selection drives

516 chemical resistance of Datura stramonium. PeerJ 4: e1898 DOI: 10.7717/peerj.1898.

517

518 Mithöfer A, Boland W. 2012. Plant defense against herbivores: chemical aspects. Annual Review

519 of Plant Biology 63:431-50 DOI: 10.1146/annurev-arplant-042110-103854.

520

521 Mondragon-Palomino M, Meyers BC, Michelmore W, Gaut BS. 2002. Patterns of positive

522 selection in the complete NBS-LRR gene family of Arabidopsis thaliana. Genome Research

523 12(9):1305-1315 DOI: 10.1101/gr.159402.

524

525 Nakajima K, Hashimoto T, Yamada Y. 1993. Two tropinone reductases with different stereo

526 specificities are short-chain dehydrogenases evolved from a common ancestor. Proceedings of

527 the National Academic of Science USA 90:9591-9595 DOI: 10.1073/pnas.90.20.9591.

528

529 Ohno S. 1970. Evolution by gene duplication. Springer-Verlag Press.

530

531 Panchy N, Lehti-Shiu M, Shiu SH. 2016. Evolution of gene duplication in plants. Plant

532 Physiology 171(4):2294-2316 DOI: 10.1104/pp.16.0052.

533

534 Picazo-Aragonés J, Terrab A, Balao F. 2020. Plant volatile organic compounds evolution

535 transcriptional regulation, epigenetics and polyploidy. International Journal of Molecular

536 Sciences 218986 DOI:10.3390/ijms21238956

537

538 Pigatto AS, Blanco CC, Mentz LA, Soares GL. 2015. Tropane alkaloids and calystegines as

539 chemotaxonomic markers in the Solanaceae. Anais de Academia Brasileira de Ciencias

540 87(4):213949.

541

542 Razali R, S. Bougouffa, Morton M, Lightfoot DJ, Alam I, Essack M, Arold S, Kamau A,

543 Schmöckel S, Pailles Y, Shaid M, Michel C. Al-Babili S, Shwen Y, Tester M, Bajic, Negrap S.

PeerJ reviewing PDF | (2020:02:45794:1:2:NEW 15 Apr 2021) 
544 2018. The genome sequence of the wild Tomato Solanum pimpinellifolium provides insights into

545 salinity tolerance. Frontiers in Plant Science 9:1402 DOI: 10.3389/fpls.2018.01402.

546

547 Seifi HS \& Shelp BJ. 2019. Spermine differentially refines plant defense responses against biotic

548 and abiotic stresses. Frontiers in Plant Science 10:117 DOI: 10.3389/fpls.2019.00117.

549

550 Shidore T, Triplett LR. 2017. Toxin-antitoxin systems: implications for plant disease. Annual

551 Review of Phytopathology 55(1):161-179 DOI: 10.1146/annurev-phyto-080516-035559.

552

553 Shonle I, Bergelson J. 2000. Evolutionary ecology of the tropane alkaloids of Datura

554 stramonium L. (Solanaceae). Evolution 54(3):778-788 DOI: 10.1111/j.0014-3820.

555 2000.tb00079. x.

556

557 Sierro, N. Battey JN, Quadi S, Boved L, Goeofert S, Bakaher N, Ivanov N.2013. Reference

558 genomes and transcriptomes of Nicotiana sylvestris and Nicotiana tomentosiformis. Genome

559 Biology14, R60 DOI: 10.1186/gb-2013-14-6-r60.

560

561 Simonsen M, Mailund T, Pedersen CNS. 2008. Rapid neighbor-joining. Lectures Notes

562 Computational Sciences 5251: 113-122 DOI: 10.1007/978-3-540-87361-7_10.

563

564 Singh B, Sharma RA. 2015. Plant terpenes: defense responses, phylogenetic analysis, regulation

565 and clinical applications. Biotechnology 5(2):129-151 DOI: 10.1007/s13205-014-0220-2.

566

567 Soltis PS, Soltis DE. 2016. Ancient WGD events as drivers of key innovations in angiosperms.

568 Current Opinion in Plant Biology 30:159-165 DOI: 10.1016/j.pbi.2016.03.015.

569

570 Stanke M, Keller O, Gunduz I, Hayes A, Waak S. Morgenstern B. 2006. AUGUSTUS: ab initio

571 prediction of alternative transcripts. Nucleic Acids Research 34(2):W435:439 DOI:

$57210.1093 /$ nar/gk1200.

573

PeerJ reviewing PDF | (2020:02:45794:1:2:NEW 15 Apr 2021) 
574 The Tomato Genome Consortium. 2012. The tomato genome sequence provides insights into

575 fleshy fruit evolution. Nature 485:635-641 (2012). https://doi.org/10.1038/nature11119

576 Schrödinger Release 2021-1: Desmond Molecular Dynamics System, D. E. Shaw Research, New

577 York, NY, 2021. Maestro-Desmond Interoperability Tools, Schrödinger, New York, NY, 2021.

578 Seifi H S, Zarei A, Hsiang T, Shelp BJ. 2019. Spermine is a potent plant defense activator 579 against gray mold disease on Solanum lycopersicum, Phaseolus vulgaris, and Arabidopsis 580 thaliana. Phytopathology. 109(8):1367-1377 DOI: 10.1094/PHYTO-12-18-0470-R.

581

582 Tholl, D. 2006. Terpene synthases and the regulation, diversity and biological roles of terpene 583 metabolism. Current Opinion in Plant Biology 9(3):297-304 DOI: 10.1016/j.pbi.2006.03.014. 584 Van de Peer Y, Mizrachi E, Marchal K. 2017. The evolutionary significance of polyploidy. 585 Nature Review Genetics 18(7):411-424 DOI: 10.1038/nrg.2017.26.

586

587 Wallace I.M, Sullivan O, Higgins D G, Notredame C. 2006. M-Coffee: combining multiple 588 sequence alignment methods with T-Coffee Nucleic Acids Research 34(6): 1692-1699 DOI: 589 10.1093/nar/gk1091.

590

591 Waterhouse A, Bertoni MB, Studer S, Tauriello G, Gumienny R, Heer FT, de Beer TA, Rempfer 592 C, Bordoli L, Lepore R, Schwede T. 2018. SWISS-MODEL: homology modelling of protein 593 structures and complexes. Nucleic Acids Research 46(W1): W296-W303. 594 595

Wink M. 2003. Evolution of secondary metabolites from an ecological and molecular 596 phylogenetic perspective. Phytochemistry 64:3-19 DOI: 10.1016/s0031-9422(03)00300-5

597

598

Xu S, Brockmöller T, Navarro-Quezada A, Kuhl H, Gase K, Ling Z, Zhou W, Kreitzer C, Stanke 599 M, Tang H, Lyons E, Pandey P, Pandey SP, Timmermann B, Gaquerel E, Baldwin IT. 2017.

600 Wild tobacco genomes reveal the evolution of nicotine biosynthesis. Proceedings of the National 601 Academy of Sciences USA 114:6133LP-LP6138 DOI: 10.1073/pnas.1700073114.

602 
603 Yang Z, Nielsen R. 2000. Estimating synonymous and nonsynonymous substitution rates under 604 realistic evolutionary models. Molecular Biology and Evolution 17(1):32-43 DOI:

605 10.1093/oxfordjournals.molbev.a026236.

606 Zhou F., Pichersky E. 2020. The complete functional characterization of the terpene synthase 607 family in tomato. New Phytologist 226(5):1341-1360 DOI: 10.1111/nph.16431.

608

609 
Figure 1

Figure 1. Phylogenetic analysis of TRI and TRII protein sequences

Phylogenetic analysis of TRI and TRII protein sequences. TR-I and TR-II define Group I and II, respectively, in Solanaceae (A). Substitution rates $(\omega)$ of TR-II (B). Three duplication events in the genome of Datura stramonium are indicated (diamonds).

A

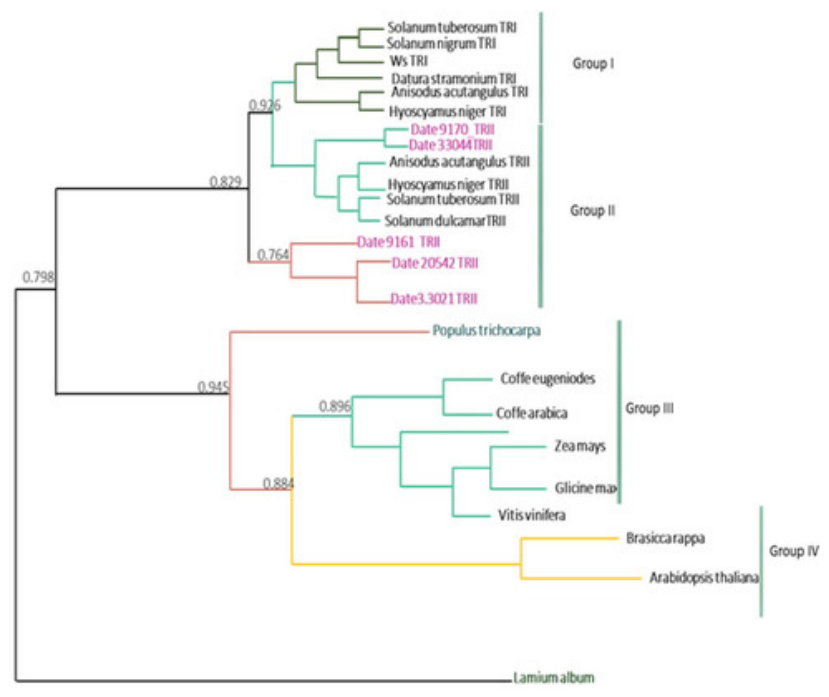

B

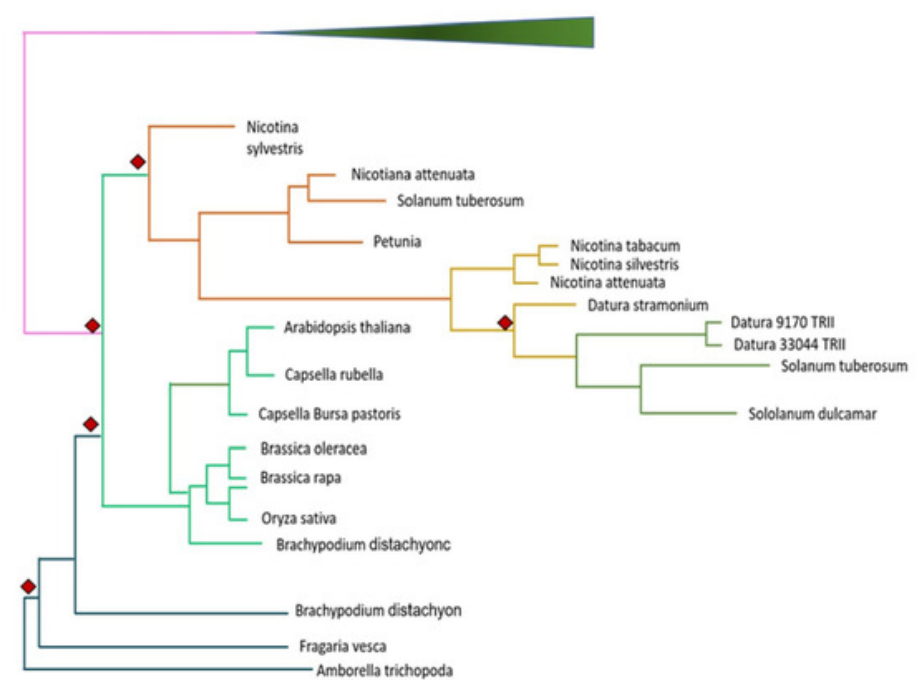


Figure 2

Figure 2. Structure of domains of TRI

Phylogenetic analysis and structure of domains of TRI gene in different species. Six copies are distributed in different clades. Tic23dati33027 has an extra domain adh_short_C.

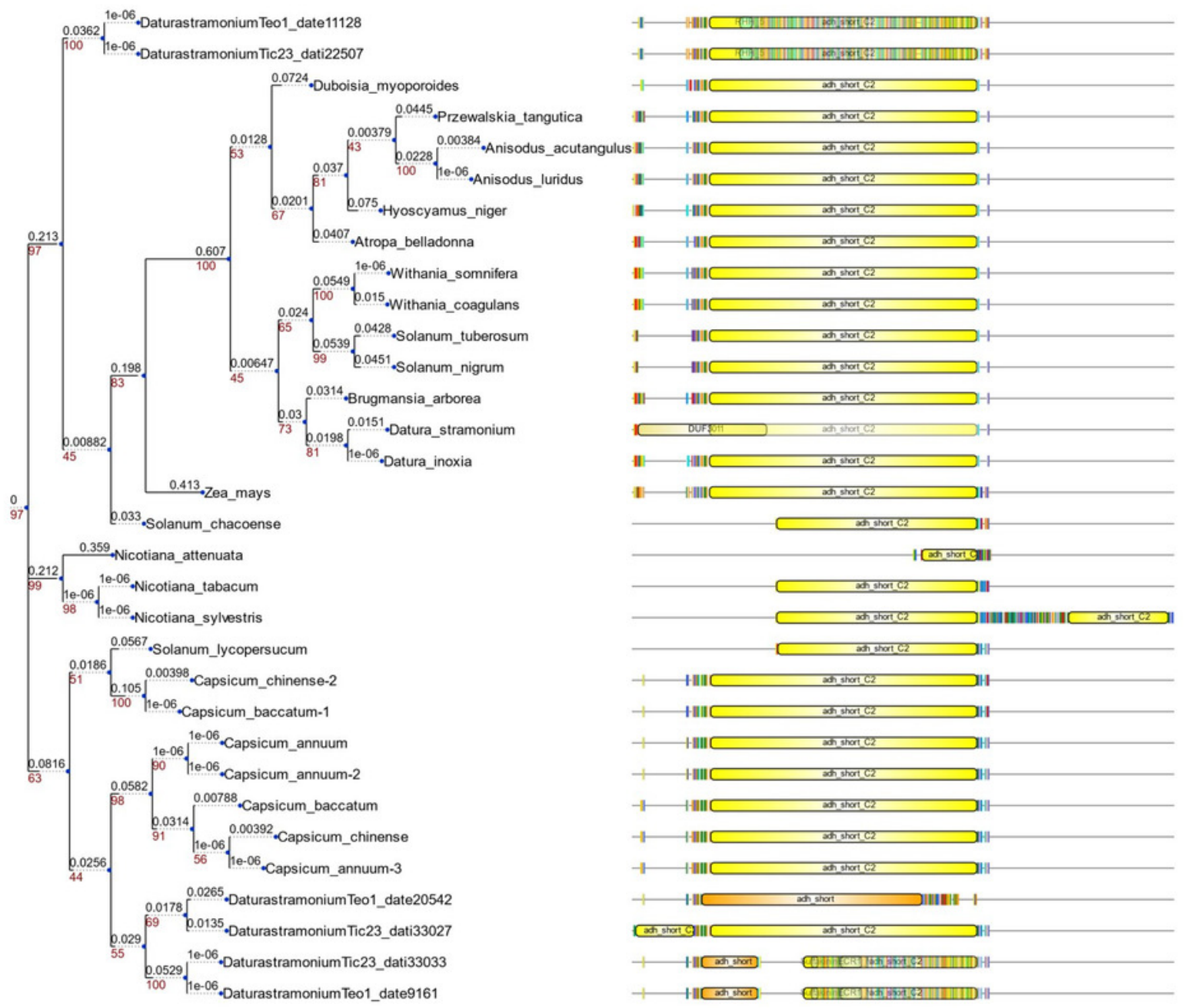




\section{Figure 3}

Phylogeny of gene $\mathrm{H6H}$

Phylogeny of gene $\mathrm{H6H}$ in 19 species. In A we observe two copies of the gene are present in the two sequenced genomes of $D$. stramonium (Tic and Teo). In Tic8550 there is a tandem duplication of dominion DIOX N (PF14226) vs. Teo 19488; two copies of the gene are present in both genomes (segmental duplication) distributed in different clades. B. Alignment of sequences of protein $\mathrm{H} 6$ with the conserved zones highlighted. In the terminal carboxyl, the DIOX-N dominion is duplicated in TIC8550.

A

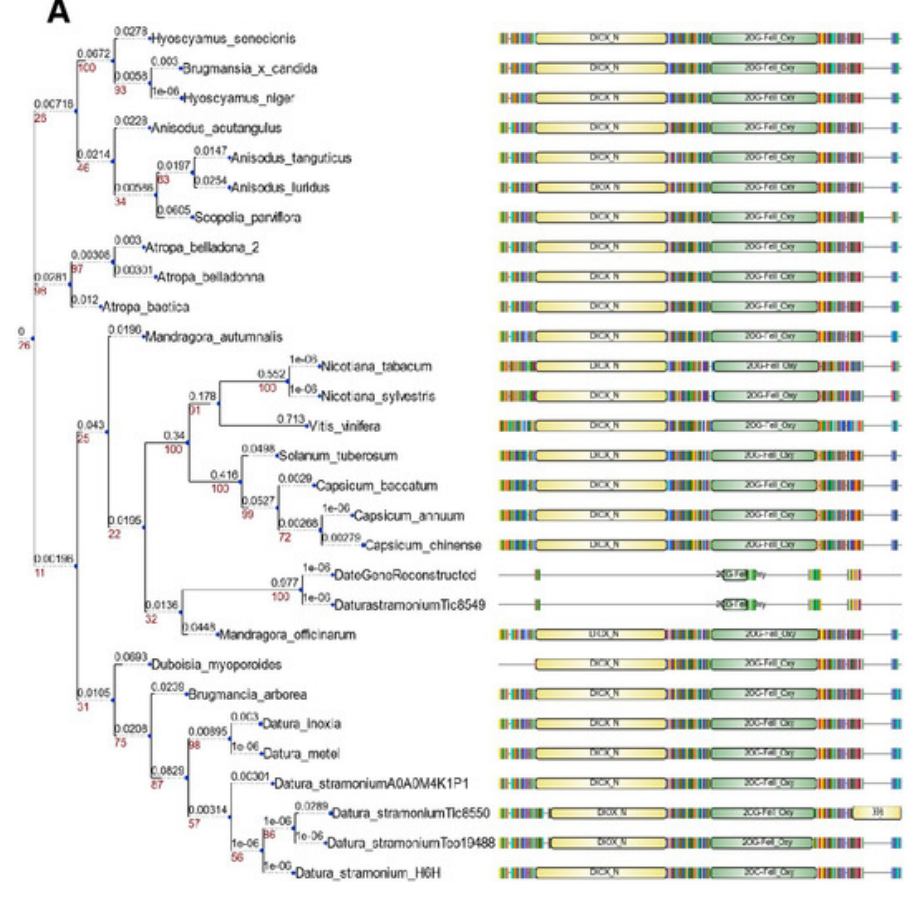

B

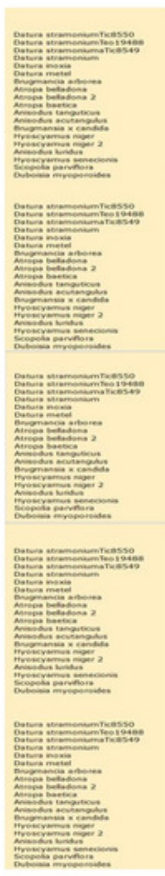

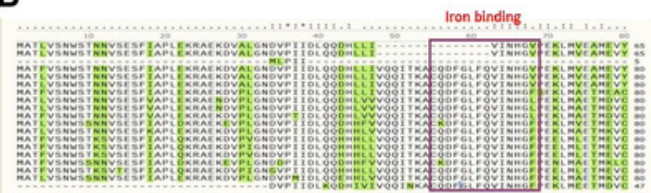

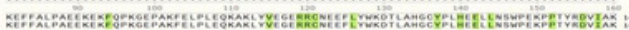
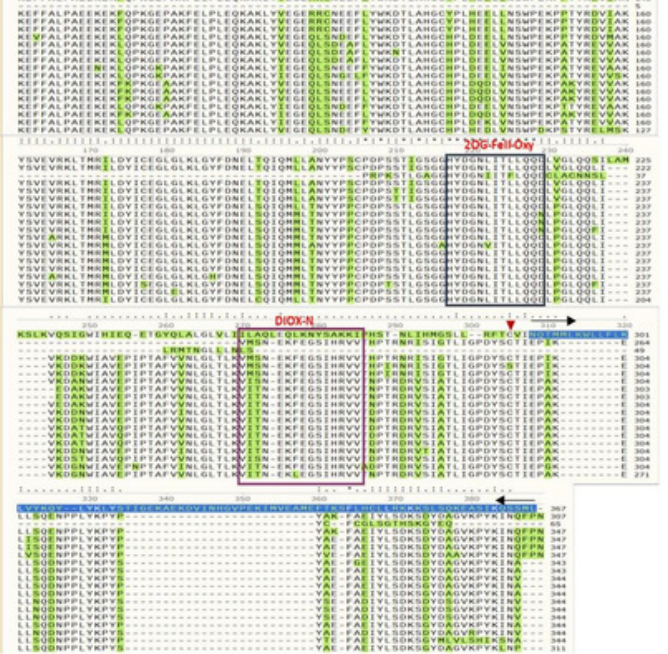


\section{Figure 4}

Phylogeny of pmt

Phylogeny of Putrescine N-methyltransferase $(p m t)$. date = Datura stramonium Teotihuacán, dati $=$ Datura stramoniumTicumán. The genomic analyses indicate an expansion of $p m t$, two copies in date and five in dati. The latter has an additional dominion of spermine synthase.

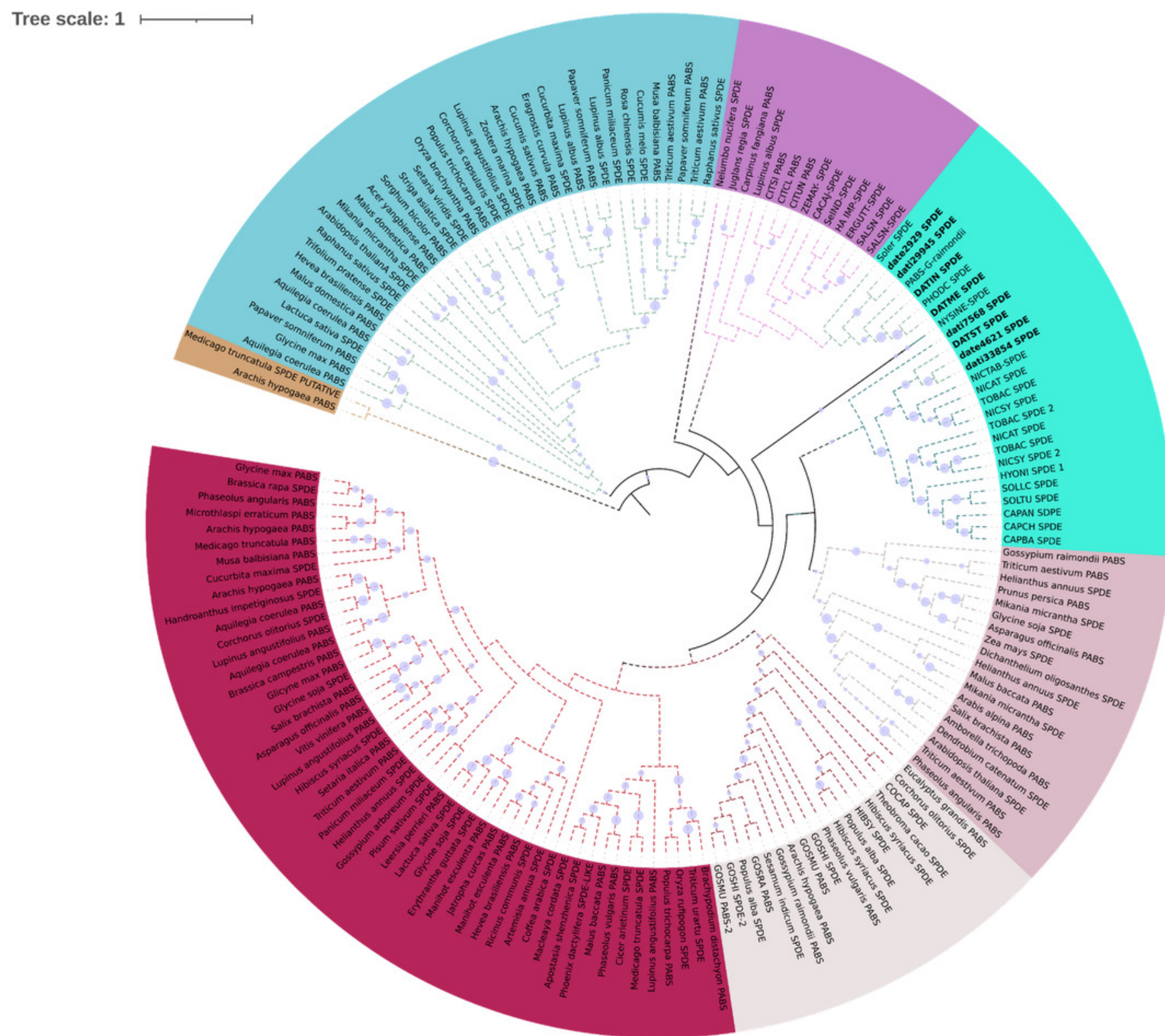




\section{Figure 5}

Phylogeny of TPS

Phylogeny of TPSs. 18 TPSs were found in D. stramonium (white letters) and Solanaceae and other angiosperms. Two copies of TPS-10 and TPS-13 in the subfamily TPSa. Tree obtained by Bayesian inference, with JTT+G+F evolutionary model. Most branches have a bootstrap support of 1 .

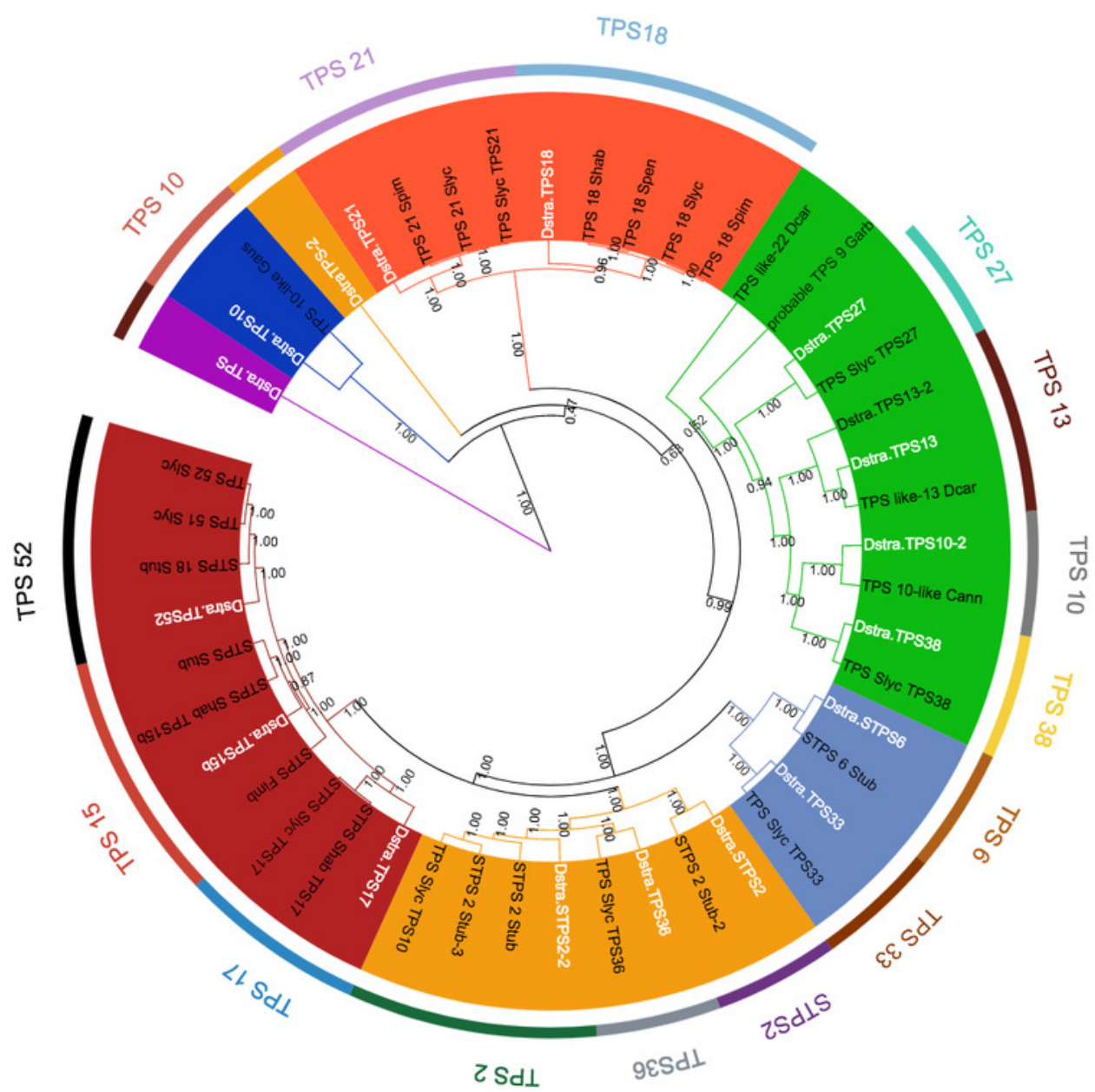




\section{Figure 6}

Phylogeny of TPS-10

Phylogeny of TPS-10 (green) of Datura stramonium, D. metel and other Solanaceae. In these species the TPS-10 is duplicated. Tree obtained by Bayesian inference, with JTT+G+F evolutionary model. Most branches have a support above $80 \%$.

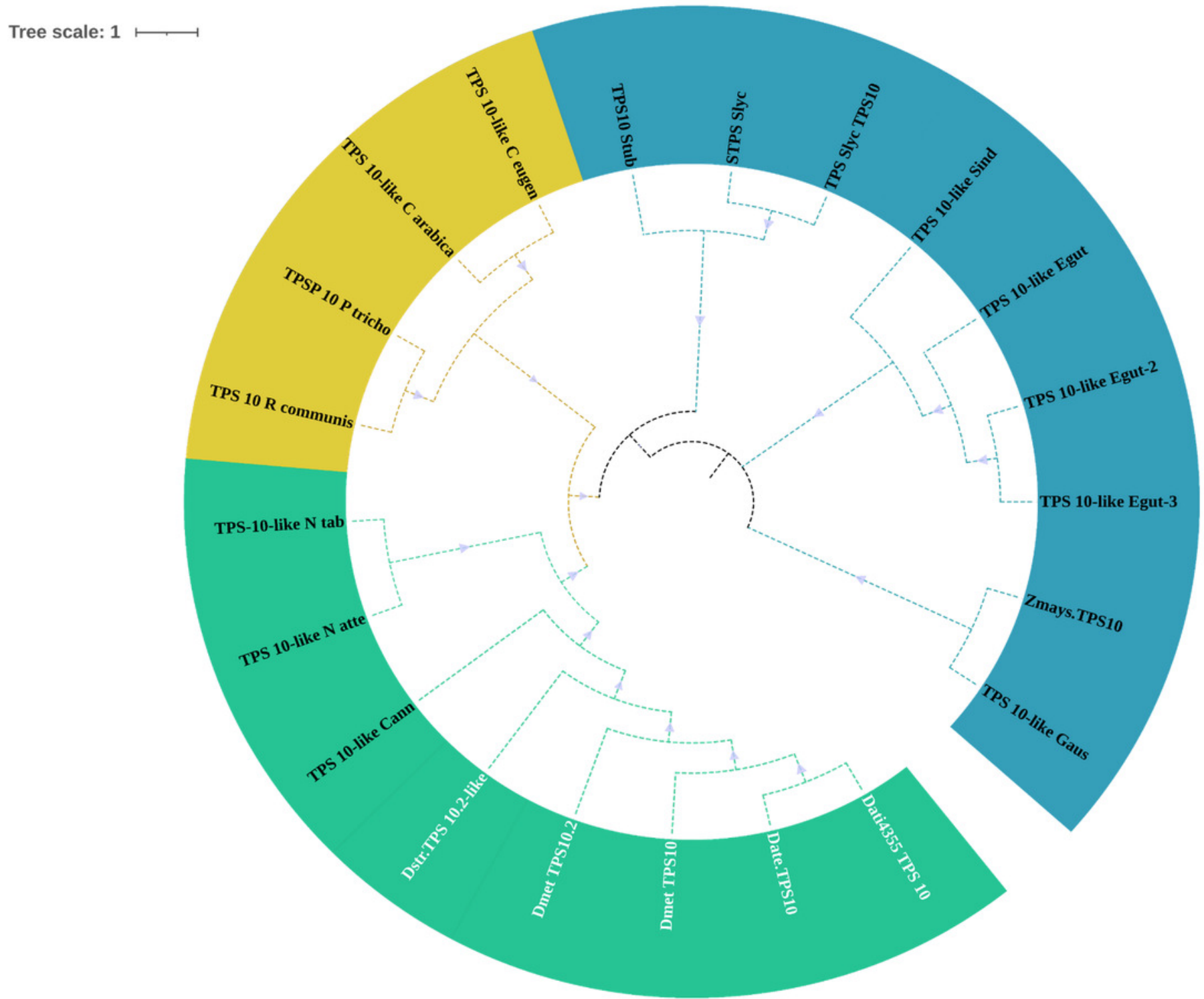


Figure 7

Structure of 6TTM in Datura metel

(A) Structure of 6TTM in Datura metel (yellow). The ligand (blue) inside the BP is surrounded by amino acids (gray). 8B) Structure modelled for Datura stramoniumTeo_19488 (cyan) and (C) D. stramonium Tic_8550. The interactions between the predicted residues of the BP and the ligand Hyo are illustrated in D, E and F.
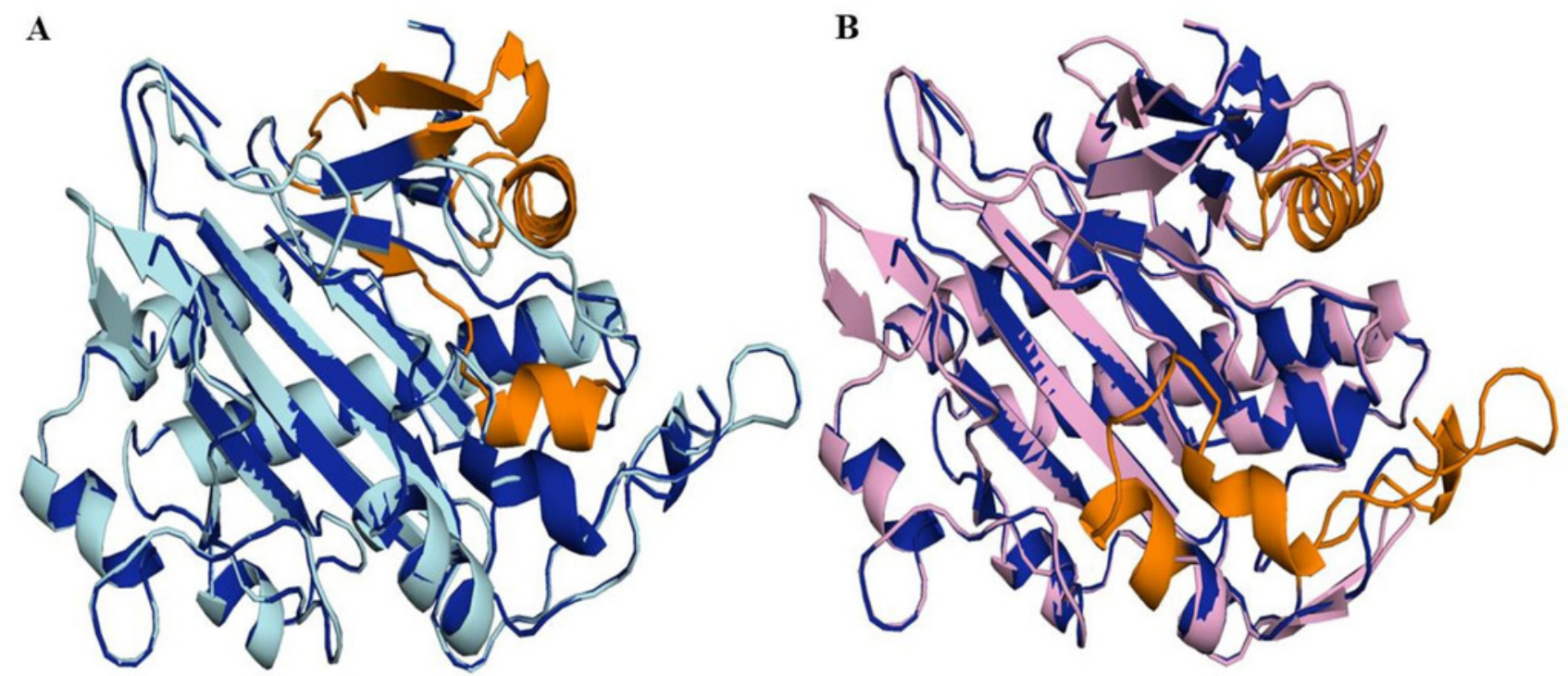


\section{Figure 8}

Models of $\mathrm{H} 6 \mathrm{H}$

Superimposition of reported structure of DmH6H (PDB ID 6TTM) (blue) and models obtained for the sequence Ticu8549 (yellow) (A) and a fragment of the sequence Ticu8550 (green)(B). Both sequences possess structural similarity with other sequences of the same protein. The fragment Ticu8549 (yellow) aligns in the region where residues of the active site of $\mathrm{H} 6 \mathrm{H}$ are located (gray) and involved in the coordination of the structure with metals (C). This site is highly conserved in the enzyme families ODD. (D) surface of fragments Ticu8549 (Yellow) and Tic8550_Fragment (green) superimposed on $\mathrm{H6H}$ structure. In red are the residues that compose the active (binding) site.

A

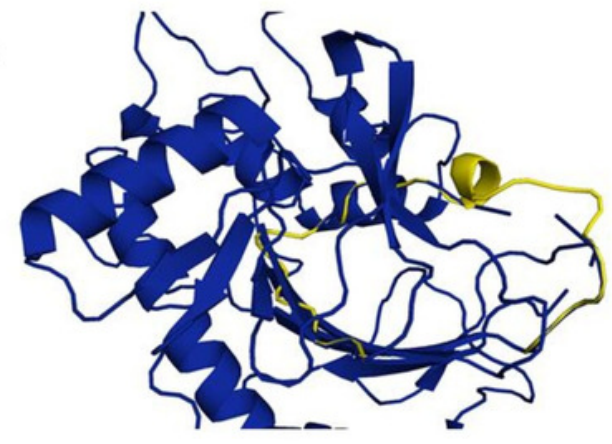

C

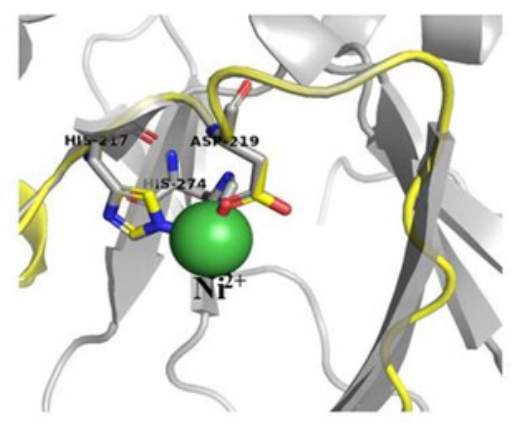

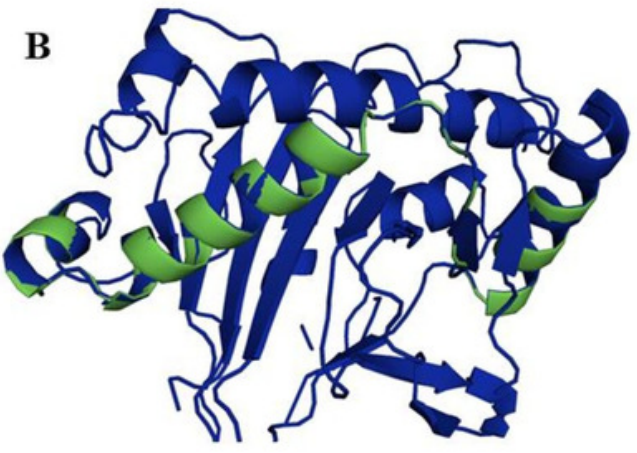

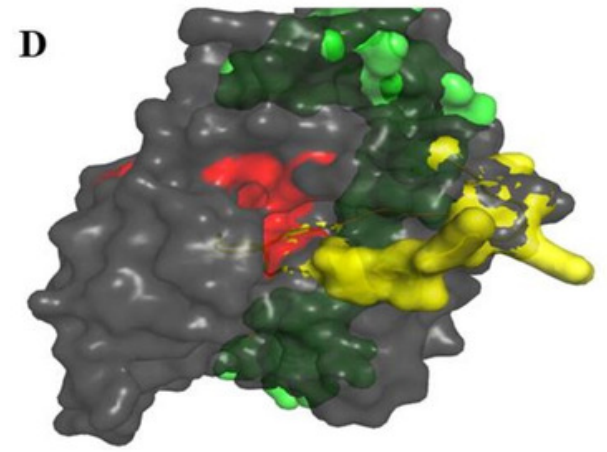


Figure 9

\section{Structure of $6 T T M$}

(A) Structure of 6TTM in Datura metel (yellow). The ligand (blue) inside the BP is surrounded by amino acids (gray). B) Structure modelled for Datura stramoniumTeo_19488 (cyan) and (C) D. stramonium Tic_8550. The interactions between the predicted residues of the BP and the ligand Hyo are illustrated in D, E and F.

A

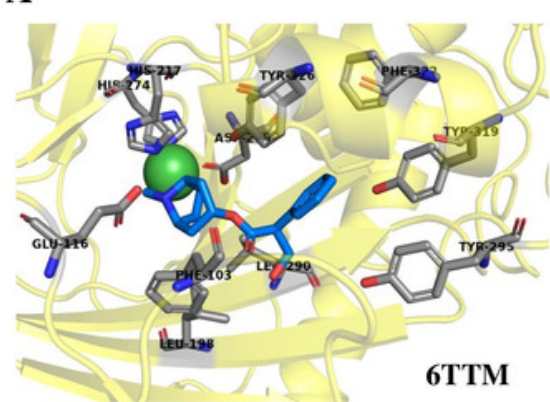

D

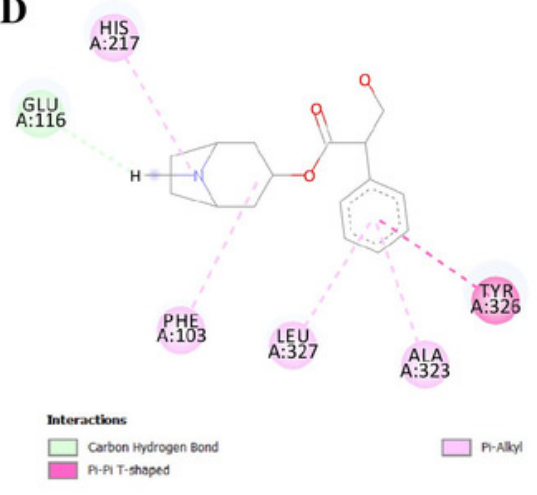

B

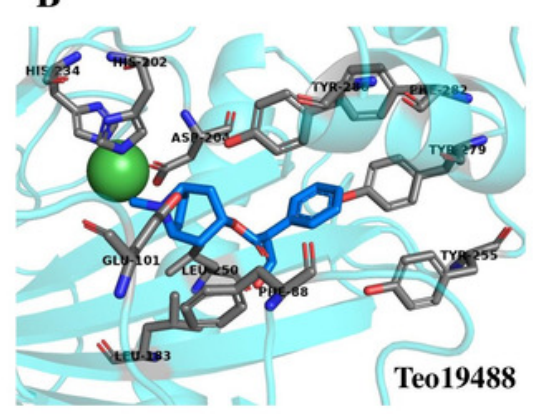

E

A:115

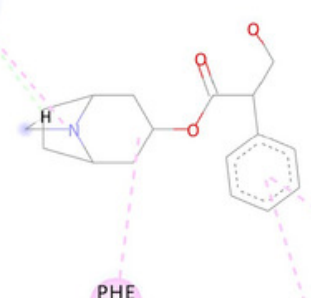

C

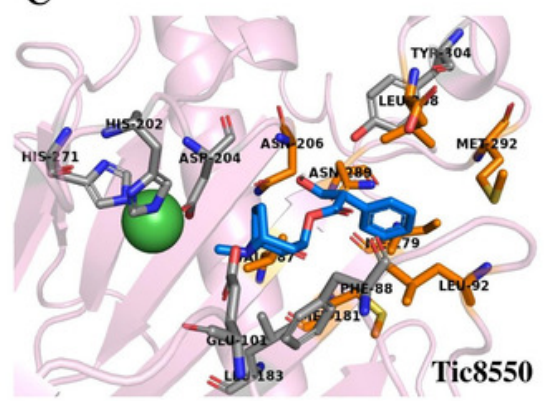

F

AEL

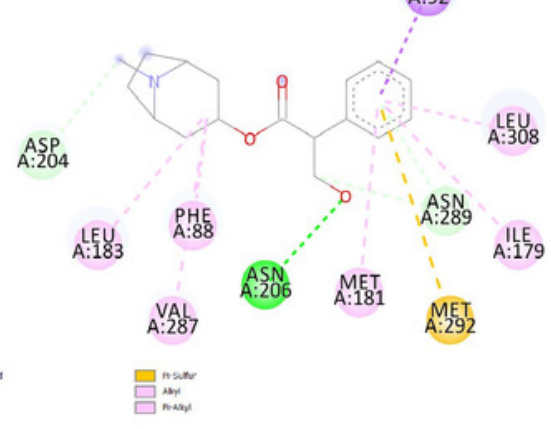




\section{Table 1 (on next page)}

Table 1

Genomes of reference employed in this study. 
Table 1. Genome of Solanaceae employed in this study

\begin{tabular}{|c|c|c|}
\hline Specie & Link & Link \\
\hline Nicotiana tabacum & https://solgenomics.net/organism/Nicotiana tabacum/genome & https://solgenomics.net/tools/blast/ \\
\hline Nicotiana sylvestris & https://solgenomics.net/organism/Nicotiana sylvestris/genome & https://solgenomics.net/tools/blast/ \\
\hline Nicotiana attenuata & https://solgenomics.net/organism/Nicotiana attenuata/genome & https://solgenomics.net/tools/blast/ \\
\hline Nicotiana tomentosiformis & https://solgenomics.net/organism/Nicotiana tomentosiformis/genome & https://solgenomics.net/tools/blast/ \\
\hline Solanum pimpinellifolium & https://solgenomics.net/organism/Solanum pimpinellifolium/genome & https://solgenomics.net/tools/blast/ \\
\hline Solanum lycopersicum & https://solgenomics.net/organism/Solanum_lycopersicum/genome & https://solgenomics.net/tools/blast/ \\
\hline Solanum pennellii & https://solgenomics.net/organism/Solanum pinnelii/genome & https://solgenomics.net/tools/blast/ \\
\hline Solanum tuberosum & $\underline{\text { https://solgenomics.net/organism/Solanum tuberosum/genome }}$ & https://solgenomics.net/tools/blast/ \\
\hline Capsicum anпиum & https://solgenomics.net/organism/Capsicum annuum/genome & https://solgenomics.net/tools/blast/ \\
\hline
\end{tabular}




\section{Table 2 (on next page)}

Table 2

Selection test for tropane alkaloids (TAs) and terpene syntahses (TPSs) genes of Datura stramonium. 
1

\begin{tabular}{|c|c|c|c|}
\hline \multirow[b]{2}{*}{ Genes } & \multicolumn{3}{|c|}{ Proportion } \\
\hline & $\begin{array}{c}\text { Chi- } \\
\text { Square }\end{array}$ & $\begin{array}{l}\text { of sites } \\
\text { selected }\end{array}$ & $\omega$ \\
\hline$P M T$ & 5.43432 & 0.1389 & 2.5778 \\
\hline$T R I$ & 0 & NA & \\
\hline TRII & 31.5335 & 0.09994 & 10.6356 \\
\hline$H 6 H$ & 15.66146 & 0.15136 & 6.469 \\
\hline TPS10_1 & 22.75614 & 0.07163 & 1.704 \\
\hline TPs10_2 & 16.16614 & 0.014589 & 2.295 \\
\hline TPS14 & 4.67532 & 0.098812 & 3.7809 \\
\hline$T P S 21$ & 1.43981 & NA & \\
\hline GGPS1 & 3.89631 & 0.0899 & 2.5988 \\
\hline
\end{tabular}

2 